\title{
Surfing on the bar: the formation of anti-truncated stellar disk profiles
}

\author{
Jakob Herpich ${ }^{1}$, Gregory S. Stinson ${ }^{1}$, Aaron A. Dutton ${ }^{1,2}$, \\ Hans-Walter Rix ${ }^{1}$ and Marie Martig ${ }^{1}$ \\ ${ }^{1}$ Max-Planck-Institut für Astronomie, Königstuhl 17, D-69117 Heidelberg, Germany \\ email: herpich@mpia.de \\ ${ }^{2}$ New York University Abu Dhabi, PO Box 129188, Abu Dhabi, UAE
}

\begin{abstract}
The stellar radial profiles of disk galaxies are often observed to be truncated, or anti-truncated in the galaxies' outskirts. As of now, the literature about galaxy formation lacks a model for the formation of observed anti-truncated stellar disks which is based on secular processes. We present an attempt to fill this gap. We were able to model anti-truncated disks in numerical SPH simulations of the formation of isolated galaxies. We will show that the stars in the outskirts of the simulated galactic disk are on very eccentric orbits but were formed on circular orbits at much smaller radii. We argue that a strong central bar is the main driver of the formation of such a disk configuration. The model predicts that such outer stellar disks should show very slow rotation, but high radial dispersion. If confirmed, their existence would constitute galaxy disks of qualitatively very new kinematic properties.
\end{abstract}

Keywords. galaxies: evolution, galaxies: kinematics and dynamics, methods: n-body simulations

\section{Introduction}

The first studies of the spatial light distribution of disk galaxies found their radial profiles to follow an exponential shape (de Vaucouleurs 1957; de Vacouleurs 1958; Freeman 1970). As observations became more sensitive, broken exponential profiles had been identified in most of the galaxies (e.g. van der Kruit 1979; Pohlen et al. 2004). These can be either truncated (e.g. Pohlen et al. 2002) or anti-truncated. The latter have first been observed by Erwin, Beckman \& Pohlen (2005).

In Herpich et al. (2015) we used hydrodynamical simulations of galaxy formation in isolated Milky-Way mass dark matter halos to reproduce the entire population of disk profile shapes. In those simulations, the type of break depends on the initial angular momentum (halo spin) of the baryonic component in the simulations. Halos with lots of initial angular momentum end up hosting galaxies with truncated disk profiles, halos with little initial angular momentum form galaxies with anti-truncated disk profiles. Pure exponential profiles only occur in halos with initial angular momentum in a narrow range between the respective regimes of broken disk profiles.

Due to the design of the simulations from Herpich et al. (2015) (isolated/noncosmological), the formation mechanism of the broken disk profiles must be secular, i. e. internal to the host halo. The formation of truncated disk profiles due to secular evolution has been achieved in simulations by Roškar et al. (2008) and in analytical models by Dutton (2009). As of now, the formation of anti-truncated profiles could only be attributed to external triggers (Younger et al. 2007; Kazantzidis et al. 2009; Roediger et al. 2012; Minchev et al. 2012; Borlaff et al. 2014). For the first time we present a purely secular mechanism that forms anti-truncated stellar disk profiles. 


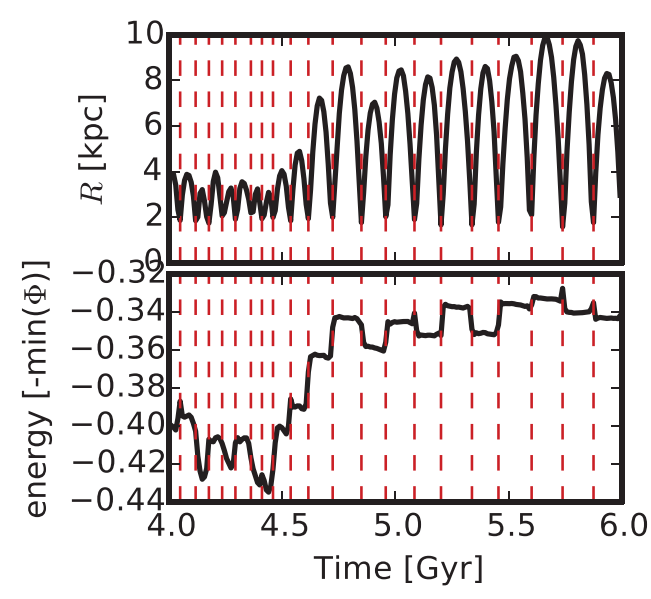

Figure 1. Orbital evolution of a stellar particle, located outside the break radius in the final simulation snapshot. The top and bottom panels show the radial position and the orbital energy of the particle respectively for a period of 4-6 Gyr into the simulation. The vertical dashed lines indicate the minima of the radius.

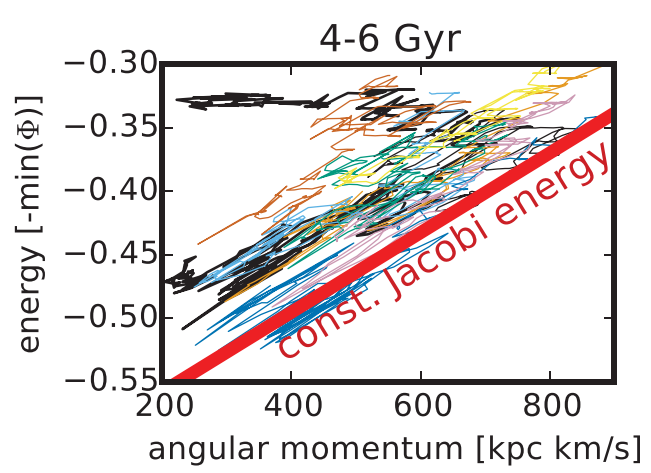

Figure 2. Stellar trajectories in the Lindblad diagram of the star from Fig. 1 (intermediate black line) and eight other randomly chosen stars, located outside the break radius in the final simulation snapshot (thin colored lines). The thick red line indicates the slope of constant Jacobi energy with respect to the galactic bar's pattern speed.

\section{Forming anti-truncations}

Here we show how the anti-truncation in one of our slowly rotating simulations is formed. All star particles that comprise the disk outside the break radius in the final simulation snapshot, are on very eccentric orbits. These stars were born at much smaller radii and on mostly circular orbits. Thus we need to understand, how these stellar orbits become eccentric and migrate to large radii.

Therefor we studied the evolution of the orbits of an individual stellar particle that is located beyond the break radius in the final simulation snapshot. In Fig. 1 we show the evolution of the radial position (top panel) and orbital energy (bottom panel) of this stellar particle between 4 and 6 Gyr into the simulation. The orbit's semi-major axis grows considerably during this period. The minimum radius stays constant for each orbit. This indicates that the star's orbit becomes more eccentric. After 4.5 Gyr the star's orbital energy changes only at pericenter and thus its evolution approximately follows a step function. Thus the star particle gains the energy necessary to migrate to large radii at the center of the simulated galaxy.

The simulations with little initial angular momentum have strong bars. In particular, they are stronger than those in the simulations with high initial angular momentum. In the particular simulation that we analysed, the bar is long lasting with a constant pattern speed $\Omega_{\mathrm{p}} \approx 100 \mathrm{~km} \mathrm{~s}^{-1} \mathrm{kpc}^{-1}$. This introduces a non-axisymmetric perturbation to the potential of the simulated galaxy, that is steadily rotating with a constant pattern speed. In such a system, the Jacobi integral (or Jacobi energy) $e_{\mathrm{J}}=e-\Omega_{\mathrm{p}} j$ is constant, where $e$ and $j$ are the specific orbital energy and angular momentum of the stellar particle. Given the constancy of $e_{\mathrm{J}}$ and $\Omega_{\mathrm{p}}$ particles trajectories, that are effected by the rotating bar in the simulations should follow a constant slope in the $e-j$ plane, also known as Lindblad diagram Binney \& Tremaine 2008. In Fig. 2 we show the trajectories in the Lindblad diagram for the star from Fig. 1 (intermediate black line) and eight other stars that have been randomly selected from those stars located outside the break radius (thin colored lines). The thick red line indicates the slope of constant Jacobi energy. We see that there 
may be some horizontal evolution (constant $e$ ) but that any change in orbital energy $e$ dominantly obeys the slope of constant Jacobi energy with respect to the rotating bar. We interpret this as a clear signature of the bar as the main driver for outward migration of practically all stars that comprise the outskirts of the stellar disk which corresponds to the shallow outer part of the broken exponential surface density profile of the simulated galaxy.

\section{Conclusion and observational predictions}

We show that anti-truncated stellar disks can be formed via a purely secular mechanism. This mechanism is based on reoccuring interactions of stellar particles with the rotating potential of a strong and long-lasting central bar. These interactions can drive a substantial radial heating of stellar orbits accompanied by a growth in semi-major axis. A substantial amount of stars experience such an orbit transformation and ultimately make up the outskirts of the anti-truncated disk in the simulated galaxies.

The dynamics of stars in the outskirts of these disks give rise to very peculiar global dynamics of the corresponding stellar disks, providing a potential observational test. The average azimuthal rotational velocity of stars is up to a factor of four slower than that expected of a rotationally supported disk comprised of stars on near circular orbits. The radial velocity dispersion of all stars in the disk component of the galaxies exceeds the value of the azimuthal and vertical velocity dispersion by almost an order of magnitude. Thus, anti-truncated disks, formed via the proposed mechanism, are predicted to be slowly rotating and anisotropic, particularly in their outskirts.

\section{References}

Binney, J. \& Tremaine, S. 2008, Galactic dynamics, 2nd ed., Princeton University Press, Princeton, NJ

Borlaff, A., Eliche-Moral, M. C., Rodríguez-Pérez, C., Querejeta, M., Tapia, T., Pérez-González, P. G., Zamorano, J., Gallego, J., \& Beckman, J. 2014, A\&A, 570, A103

de Vaucouleurs, G. 1957, $A J, 62,96-82$

de Vaucouleurs, G. 1958, ApJ, 128, 465

Dutton, A. A. 2009, MNRAS, 396, 121-140

Erwin, P., Beckman, J. E., \& Pohlen, M. 2005, ApJ (Letters), 626, L81-L84

Freeman, K. C. 1970, ApJ, 160, 811

Herpich, J., Stinson, G. S., Dutton, A. A., Rix, H.-W., Martig, M., Roškar, R., Macciò, A. V., Quinn, T. R., Wadsley, J. 2015, MNRAS, 448, L99-L103

Kazantzidis, S., Zentner, A. R., Kravtsov, A. V., Bullock, J. S., \& Debattista, V. P. 2009, ApJ, $700,1869-1920$

Minchev, I., Famaey, B., Quillen, A. C., Di Matteo, P., Combes, F., Vlajić, M., Erwin, P., \& Bland-Hawthorn, J. 2012, A\&\&A, 548, A126

Pohlen, M., Beckman, J. E., Hüttemeister, S., Knapen, J. H., Erwin, P., \& Dettmar, R.-J. 2004, in: Block, D. L.; Puerari, I.; Freeman, K. C.; Groess, R. \& Block, E. K. (eds.), Penetrating Bars Through Masks of Cosmic Dust, 319, 713

Roediger, J. C., Courteau, S., Sánchez-Blázquez, P., \& McDonald, M. 2012, ApJ, 758, 41

Roškar, R., Debattista, V. P., Stinson, G. S., Quinn, T. R., Kaufmann, T., \& Wadsley, J. 2008, ApJ (Letters), 675, L65-L68

van der Kruit, P. C. 1979, A\& $A S$, 38, 15-38

Younger, J. D., Cox, T. J., Seth, A. C., \& Hernquist, L. 2007, ApJ, 670, 269-278 